\title{
Sub-picosecond X-rays from CEBAF at Jefferson Lab.
}

\author{
Alex Bogacz , Jim Boyce*, Jim Clarke ${ }^{\dagger}$, David Douglas ${ }^{*}$, Andrew Hutton ${ }^{*}$, \\ Geoffrey Krafft ${ }^{*}$, Lia Merminga ${ }^{*}$, George Neil ${ }^{*}$, Mike Poole ${ }^{\dagger}$, Sue Smith ${ }^{\dagger}$, Vic \\ Suller ${ }^{\dagger}$ and Gwyn Williams*
}

* Jefferson Lab, 12000 Jefferson Avenue, Newport News, VA 23606, USA

${ }^{\dagger}$ CLRC Daresbury Laboratory, Warrington, Cheshire WA4 4AD, UK

\begin{abstract}
A high brightness sub-picosecond x-ray source can be created by installing an undulator at Jefferson Lab's CEBAF, a nuclear physics electron accelerator. Although the beam current is only 100 microamps, the electron beam has an extremely small emittance and energy spread, with the result that one can produce x-ray beams tunable over the range $5-30 \mathrm{keV}$ with an average brightness quite comparable to beamlines at the Advanced Photon Source (APS) at Argonne National Lab. In addition, with rms bunch lengths measured down to 85 fsecs, peak brightness values are much higher than at the APS. Furthermore, this x-ray source has similar emittance in both horizontal and vertical directions, (a socalled round beam) making it of very high potential for many applications. In order to determine if indeed such a source is worth pursuing we present "tuning curve" calculations of peak and average flux and brightness for an undulator on CEBAF. They are compared with similar calculations for a dipole and for undulator-A at the APS. Finally we calculate the impact of such a device on the CEBAF beam itself itself and find it to be much smaller than the natural energy spread of the beam.
\end{abstract}

\section{INTRODUCTION}

CEBAF is an electron accelerator whose pulse lengths are in the sub-picosecond regime $(<100 \mathrm{fs}$ fwhm may be possible). Such pulses are therefore much shorter than the typical 100ps regime of storage rings used as light sources. One of the co-authors has previously suggested making use of the synchrotron radiation emitted by the dipole bends in CEBAF[1]. Due to the low average current (100 microamps), however, the emission is 1/1000 of that typically used at synchrotron radiation facilities. In principle a brighter source could be made using an undulator rather than a dipole[1]. We have examined the lattice and determined that an undulator could in principle be placed in a 15 meter straight section at the end of the North Linac, just before arc 9. A layout of CEBAF showing the position of the undulator is displayed in Fig. 1.

\section{PARAMETERS}

At the insertion point in the CEBAF lattice the beta functions are changing according to a FODO focusing arrangement with no waist at the middle of the drift and we have taken values at the midpoint of the straight. The electron beam energy at this point under standard operating conditions is $3645 \mathrm{MeV}$, the beta functions 35 meters (both).

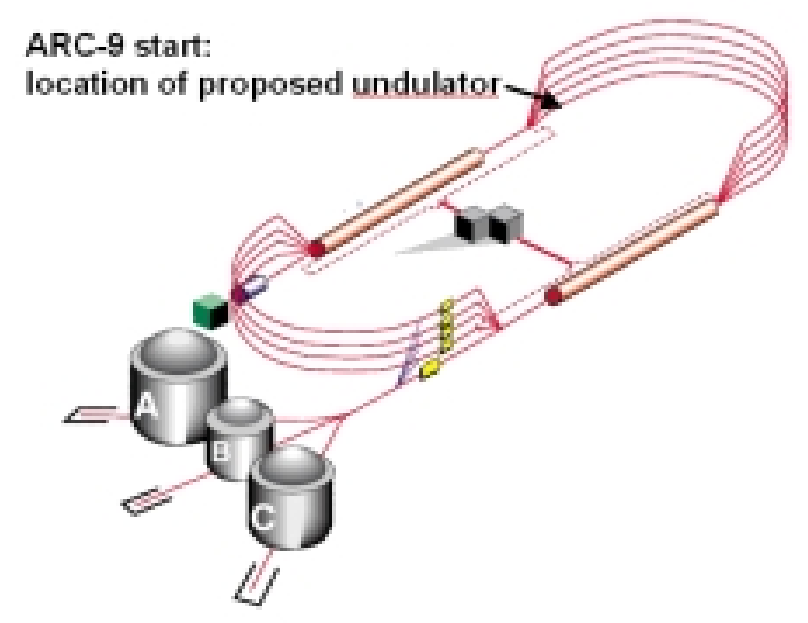

FIGURE 1. Layout of the CEBAF accelerator showing the location of the start of ARC-9. 
Furthermore, with the present FODO optics $\alpha_{\mathrm{x}}=-\alpha_{\mathrm{y}}=2.5$ giving $\sigma_{\mathrm{x}}=\sigma_{\mathrm{y}}=49$ microns and $\sigma_{\mathrm{x}}{ }^{\prime}=\sigma_{\mathrm{y}}{ }^{\prime}=3.8$ micro radians.

For the undulator we used a 70 period device with a period length of $28 \mathrm{~mm}$ and a minimum gap of $10 \mathrm{~mm}$. This undulator was chosen so that the 3rd harmonic starts at just below $5 \mathrm{keV}$ and has a maximum $\mathrm{K}$ value of 1.907, with a corresponding (peak) field of $0.7293 \mathrm{~T}$. The table below lists some key parameters:

\begin{tabular}{|l|c|c|}
\hline TABLE 1. Parameters used in the calculations. \\
\hline Parameter & APS & CEBAF \\
\hline Beam Energy (GeV) & 7 & 3.5 \\
\hline Repetition Frequency $(\mathrm{MHz})$ & 350 & 500 \\
\hline Beam Current (milliamps) & 100 & 0.1 \\
\hline $\begin{array}{l}\text { Natural (geometric) Emittance } \\
\begin{array}{l}\text { Normalized Emittance } / \gamma(\mathrm{rms}, \\
\text { nanometer radians) }\end{array}\end{array}$ & 8.2 & 0.07 \\
\hline Energy Spread (rms) & & \\
\hline Source Size Horizontal (rms, microns) & $1 \mathrm{e}-3$ & $2 \mathrm{e}-5$ \\
\hline Source Size Vertical (rms, microns) & 103 & 49 \\
\hline
\end{tabular}

\section{CALCULATIONS}

The calculations were made for the selected undulator and for both a dipole and undulator-A at the APS. The latter were chosen for comparison purposes as having well known characteristics. Currents of 100 microamps were

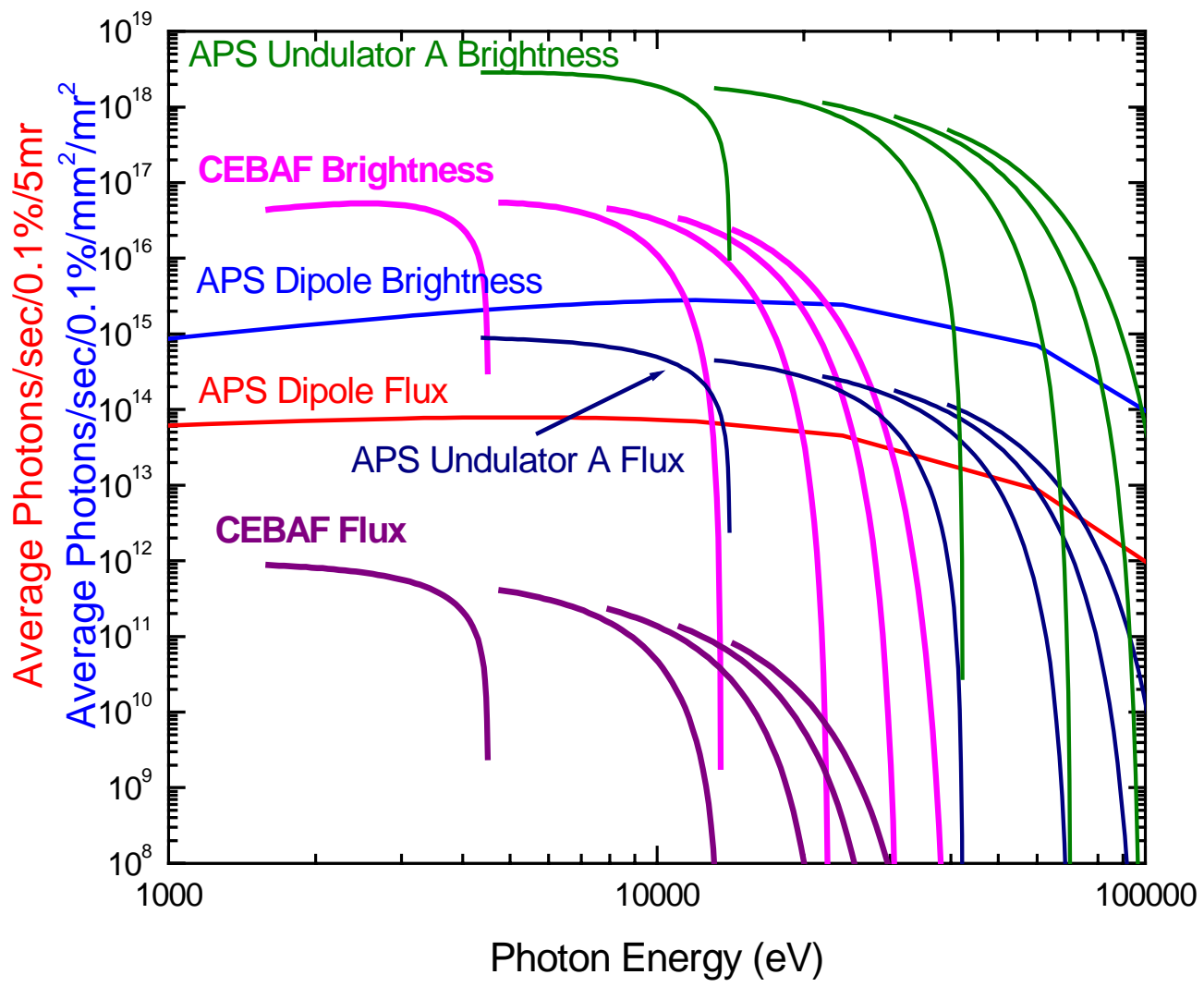

FIGURE 2. Average flux and brightness for 1. APS dipole; 2. APS undulator-A (100 milliamps); and 3. CEBAF undulator (100 microamps). Undulator tuning curves are shown for $1^{\text {st }}, 3^{\text {rd }}, 5^{\text {th }}, 7^{\text {th }}$ and $9^{\text {th }}$ harmonics (100 microamps).

used for CEBAF and 100 milliamps for the APS. Data are presented for both average and peak flux and brightness 
and are shown in Figs. 2 and 3. Calculations were repeated for odd harmonics up to the $9^{\text {th }}$, and tuning curves were generated by varying the $\mathrm{K}$ value of the undulator.

The calculations of flux are in photons/sec/0.1\% bandwidth and are into horizontal angles of 5 milliradians for the APS and $\ll<1$ milliradian for the undulators. The calculations of brightness are in units of photons $/ \mathrm{sec} / 0.1 \%$ bw $/ \mathrm{mm}^{2} / \mathrm{mrad}^{2}$ and were done with own codes. We note that the repetition frequency of each of 3 beams in CEBAF is $500 \mathrm{MHz}$, and we assumed only one beam. The frequency of the APS is $352 \mathrm{MHz}$. For the peak values we multiplied the average values by assuming $100 \mathrm{ps}$ fwhm pulses every $2.9 \mathrm{~ns}$ for the APS $(\times 29)$ and $200 \mathrm{fs}$ fwhm pulses every 2 ns for CEBAF $(\times 1 \mathrm{e} 5)$.

Under these assumptions, the average brightness obtained from CEBAF is about $6 \times 10^{16}$ photons $/ \mathrm{sec} / 0.1 \% / \mathrm{mm}^{2} / \mathrm{mrad}^{2}$, a factor of about 30 higher than that from an APS dipole and 50 times lower than an APS undulator, while the peak brightness is about $5 \times 10^{20}$ photons/ sec/ $0.1 \% / \mathrm{mm}^{2} / \mathrm{mrad}^{2}, 10$ times brighter than an APS undulator and about 4 orders of magnitude higher than from the APS dipole

Finally we comment on the effect of such an undulator on the electron beam in terms of emittance and energyspread degradation. The calculations show that the undulator extracts about 4 watts from a beam, which at 100

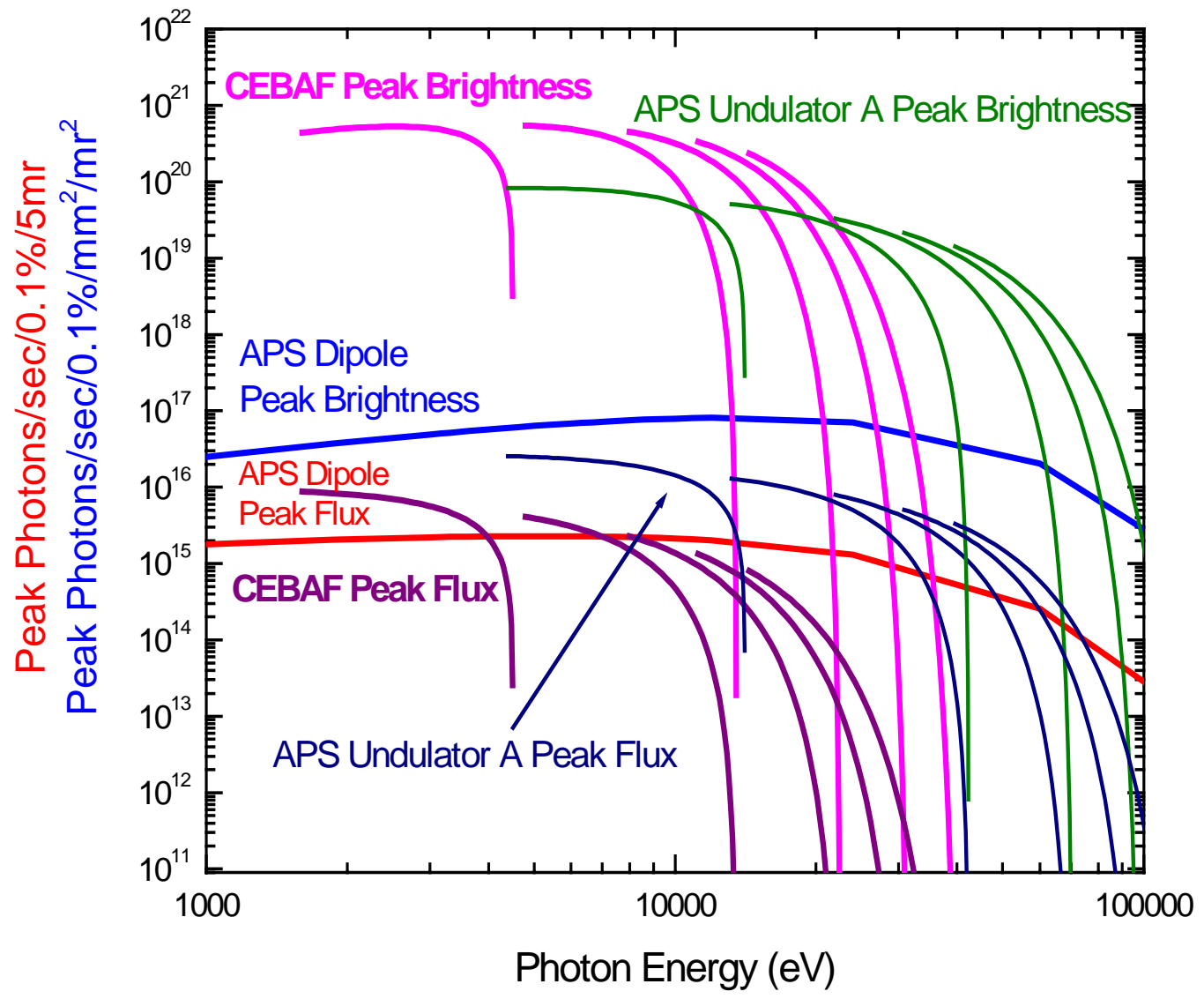

FIGURE 3. Peak flux and brightness for 1. APS dipole; 2. APS undulator-A (100 milliamps); and 3. CEBAF undulator (100 microamps). Undulator tuning curves are shown for $1^{\text {st }}, 3^{\text {rd }}, 5^{\text {th }}, 7^{\text {th }}$ and $9^{\text {th }}$ harmonics.

microamps and $4 \mathrm{GeV}$, contains 400 kilowatts. The induced energy spread is roughly twice the average loss or $2 \times 10^{-5}$ (full spread), which is a small effect when added in quadrature to the nominal rms energy spread of $2.5 \times 10^{-5}$. 


\section{CONCLUSIONS}

It is clear that an undulator on CEBAF may offer an attractive source for sub-picosecond x-ray scattering experiments with a capability of high on-sample photon fluxes. Indeed the values reported here can be increased if CEBAF is run at higher energies, and routine running is done at $5200 \mathrm{MeV}$ in the region proposed for the undulator. Unlike the situation for storage rings, the emittance will be adiabatically damped (proportional to $1 / \gamma$ where $\gamma$ is the ratio of the particle mass to its rest mass) at the higher energy and hence will be smaller.

We further note that this is a quasi-cw high repetition rate source, which is ideal for the majority of experiments. It is also mandatory for co-incidence experiments because it maximizes the ratio of true to accidental counts, the very reason for the design of CEBAF in fact. Additionally we note that this source could in principle be synchronized with Jefferson Lab's Free Electron Laser facility which offers sub-picosecond tunable beams at 75 $\mathrm{MHz}$ in the range 0.25 to 15 microns with up to 100 microjoules per pulse. This makes pump-probe dynamical experiments possible at the femtosecond frontier.

Iif these calculations indicate that CEBAF could provide an attractive sub-picosecond x-ray source, then the logistics of operation have to be carried out, and particularly location of x-ray facilities. This, together with further details of the effect of such a device on the halo and emittance of the CEBAF beam, will require a further study, which is beyond the scope of this paper. Preliminary analysis indicates a number of possible solutions.

\section{ACKNOWLEDGMENTS}

This work was supported by the U.K. Science and Engineering Research Council and by the U.S. Department of Energy under contract DE-AC05-84-ER40150.

\section{REFERENCES}

1. G. Krafft "Short Pulse Synchrotron Light from Jefferson Lab's Nuclear Physics Accelerator", Proceedings of the 1999 Particle Accelerator Conference, pgs. 2448-2450. 